\title{
Can the visual molecular configuration in computer simulations locate solid-fluid phase boundaries? The case of $\mathrm{C}_{60}$
}

\author{
M. Hasegawa ${ }^{a)}$ \\ Department of Materials Science and Technology, Faculty of Engineering, Iwate University, \\ Morioka 020-8551, Japan \\ K. Ohno ${ }^{\text {b) }}$ \\ Institute for Materials Research, Tohoku University, Sendai 980-8577, Japan
}

(Received 8 May 2000; accepted 14 June 2000)

\begin{abstract}
The Monte Carlo method is used to explicitly show that solid-fluid phase boundaries determined by the visual appearance and disappearance of a solidlike structure is quite different from that expected in the bulk system and predicted by a full free-energy analysis. This feature of the computer simulations is almost self-evident and may be understood by the argument that the phase coexistence in the finite system is inevitably suppressed by the non-negligible interfacial energy compared with that in the infinite (bulk) system. One of the discrepancies among the recent simulation studies for the high-temperature phase behavior of a model $\mathrm{C}_{60}$ is found to be the consequence of overlooking the above feature of computer simulations. (c) 2000 American Institute of Physics. [S0021-9606(00)51434-2]
\end{abstract}

\section{INTRODUCTION}

Molecular dynamics (MD) and Monte Carlo (MC) methods are the most important techniques in the simulation studies of many-body systems. These methods yield a thermodynamic average of physical quantities that are explicit functions of the phase-space coordinates of a system, such as potential energy, temperature, and pressure. However, they cannot directly yield properties that are determined by the total phase-space volume accessible to the system, entropy or (Helmholtz) free energy being such an example. Calculation of the free energy constitutes a basic problem in the study of phase transition as well as other equilibrium thermodynamic properties and requires extensive simulations over a wide range of thermodynamic parameter or along the path connecting reference and true systems. ${ }^{1}$ Efficient implementation of such a method is one of the most important issues in computer simulations. ${ }^{2}$

Another approach to the phase transition is to locate phase boundaries by the direct observation of a characteristic feature indicative of a phase coexistence in the course of changing thermodynamic parameters such as temperature and volume. The solid-fluid transition is most clearly observed through the appearance and disappearance of an ordered solidlike structure. However, the finite-size effect on the onset of phase coexistence is substantial in the usual computer simulations and the resulting phase boundaries could be quite different from those expected in the bulk system. Consider the formation of a solid nucleus in a fluid in the course of compression or lowering temperature. In finite systems the interfacial energy associated with such a nucleation of only several particles cannot be negligible, which

\footnotetext{
${ }^{a}$ Electronic mail: hasegawa@iwate-u.ac.jp

b) Present address: Department of Physics, Faculty of Engineering, Yokohama National University, Yokohama 240-8501, Japan.
}

implies that the tendency of phase coexistence is suppressed (compared with that in bulk system) and the system could remain in a single phase even if the condition of solid-fluid coexistence is satisfied in the bulk system. Consequently, the "freezing line" determined by the visual appearance of a solidlike structure would fall at higher densities (or lower temperatures) than the true (bulk) one. All these features in the computer simulations are almost self-evident and it is essential to avoid an explicit coexistence in the study of any phase transition. The Gibbs-ensemble MC (GEMC) has in fact been devised as one of such methods and provided a useful means in the study of the liquid-vapor transition. ${ }^{3}$ However, if the solid phase is involved in the phase transition, we have a practical difficulty in using the GEMC. ${ }^{1}$ We note that the finite-size effect in computer simulations is much less serious when one is dealing with a single phase not too close to the critical point, which enables one to perform accurate computations of the thermodynamic properties of each phase involved in the phase transition and thereby to locate the phase boundaries.

In the present short paper we are specifically concerned with the high-temperature phase diagram of $\mathrm{C}_{60}$, for which the above features in the computer simulations have been overlooked in some of the previous studies. Since the discovery ${ }^{4}$ of an efficient synthesis of $\mathrm{C}_{60}$ its phase behavior has attracted much attention with regard to the possibility that this substance does not have a thermodynamically stable liquid phase. ${ }^{5}$ Motivated by this possibility, several simulations $^{6-10}$ and theoretical calculations ${ }^{11-15}$ have been performed. Hagen et $a l^{6}$ and Cheng et $a l^{7}$ were the first to have performed simulations but these authors reached different conclusions; the former used a GEMC method and found that $\mathrm{C}_{60}$ has no liquid phase, whereas the latter used a MD method and predicted the existence of a stable liquid phase between $\sim 1800 \mathrm{~K}$ and $\sim 1900 \mathrm{~K}$. Both of these authors used 
the same intermolecular potential proposed by Girifalco, ${ }^{16}$ but Hagen et al. truncated this potential at $r=2 \sigma$, where $\sigma$ is the diameter of the $C_{60}$. molecule, which explains part of the discrepancies between the two simulations. In fact, the effect of such a potential truncation on the phase behavior is substantial and the use of the full potential leads to a different conclusion as to the existence of a stable liquid phase. ${ }^{15} \mathrm{We}$ also note that in their MD simulations Cheng et al. ${ }^{7}$ determined the solid-fluid phase boundaries by the visual appearance and disappearance of a solidlike structure. Such results could be different from the true (bulk) one as we have discussed in the above. The same statement also applies to the work of Abramo and Coppolino, ${ }^{17}$ who also used a MD method and located the freezing points of $\mathrm{C}_{60}$ at two temperatures by directly observing molecular configurations. The purpose of the present work is to explicitly show that the solid-fluid phase boundary determined by the visual method in computer simulations is quite different from that expected for the infinite (bulk) system and actually obtained by a full free-energy analysis.

\section{OUTLINE OF THE SIMULATIONS}

The Girifalco potential is useful for the orientationally disordered phase of $\mathrm{C}_{60}$ (Ref. 18) and has been used in most simulations and theoretical calculations for its hightemperature phase diagram. In the present work we also used the Girifalco potential, which is given by ${ }^{16}$

$$
\begin{aligned}
\phi(r)= & -A\left[\frac{1}{x(x-1)^{3}}+\frac{1}{x(x+1)^{3}}-\frac{2}{x^{4}}\right] \\
& +B\left[\frac{1}{x(x-1)^{9}}+\frac{1}{x(x+1)^{9}}-\frac{2}{x^{10}}\right],
\end{aligned}
$$

where $x=r / \sigma, \sigma$ being the diameter of the sphericalized $\mathrm{C}_{60}$ molecule as before. The parameters in Eq. (1) have been determined empirically ${ }^{16}$ and given by $A=74.94$ $\times 10^{-15} \mathrm{erg}$ and $B=135.95 \times 10^{-18} \mathrm{erg}$.

We employed a constant- $N V T$ ensemble MC method and performed simulations for the system of $N$ molecules in the cubic cell subject to the periodic boundary condition. Most simulations were performed at $N=256$ and the results refer to this case unless otherwise stated. The details of the simulation method is essentially the same as that used in previous work, ${ }^{10}$ in which an extensive free-energy analysis was made to explore the high-temperature phase diagram of $\mathrm{C}_{60}$. In the present simulations complementing the previous work we -monitored the virial pressure, configurational energy, and particle configuration. The virial pressure is given by

$$
p=\rho k_{B} T\left\{1-\frac{1}{3 N}\left\langle\sum_{i<j} r_{i j} \frac{\partial \beta \phi\left(r_{i j}\right)}{\partial r_{i j}}\right\rangle\right\},
$$

where $\rho=N / V$, particle number density, $k_{B}$ is the Boltzmann constant, $\beta^{-1}=k_{B} T$, and $\langle\cdots\rangle$ represents thermal average. The configurational energy per particle is given by

$$
u_{\mathrm{ex}}=\frac{1}{N}\left\langle\sum_{i<j} \phi\left(r_{i j}\right)\right\rangle \text {. }
$$

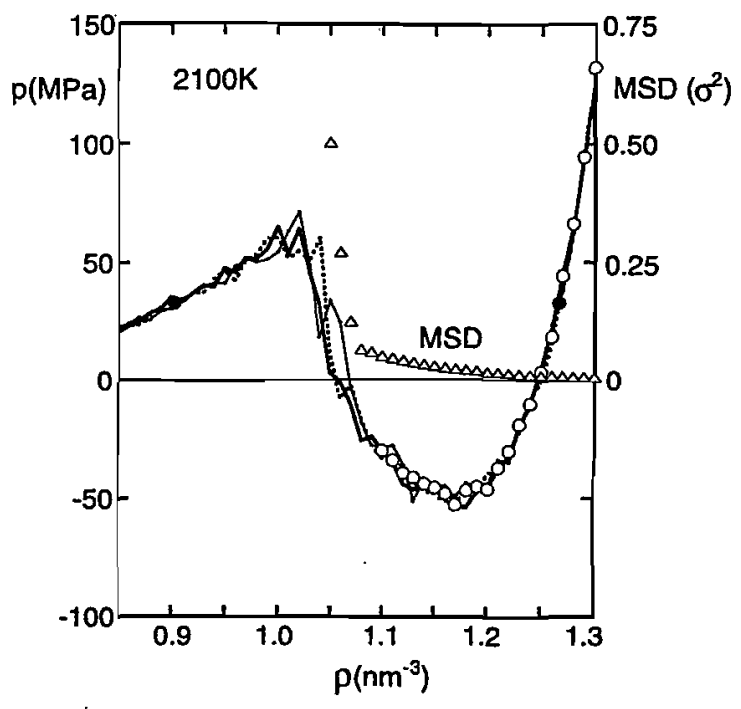

FIG. 1. MC simulation results for the virial pressure and the mean-square displacement (MSD) of $\mathrm{C}_{60}$ at $2100 \mathrm{~K}$. The thin, thick, and dotted lines show the virial pressure obtained by three different sets of runs, each starting with a fluid phase at $0.85 \mathrm{~nm}^{-3}$. The open circles show the virial pressure obtained along the step-wise decreasing densities starting with a fcc solid at $1.30 \mathrm{~nm}^{-3}$, and the triangles represent the corresponding MSD in unit of $\sigma^{2}, \sigma$ being the diameter of $\mathrm{C}_{60}$ molecule. The solid circles are the solid-fluid phase boundaries obtained by a full free-energy analysis (Ref. 10).

The slope of $u_{\mathrm{ex}}$ as. a function of temperature or density is very sensitive to the change in phase behavior and may be used as a useful indicator of a possible phase transition. ${ }^{7,17}$

\section{RESULTS AND DISCUSSION}

Figure 1 illustrates typical examples of the present MC result for the virial pressure and mean-square displacement (MSD) of $\mathrm{C}_{60}$ at $2100 \mathrm{~K}$. The virial pressure was obtained by three different runs starting with a fluid phase at $\rho$ $=0.85 \mathrm{~nm}^{-3}$. The density was increased step-wise, the increment being $\Delta \rho=0.01 \mathrm{~nm}^{-3}$ at each step, and the data of $1 \times 10^{4}$ Monte Carlo steps (MCS), i.e., $2.56 \times 10^{6}$ steps for $N=256$, were used to obtain the thermal average after 200 MCS equilibration run at each density. The calculated pressure shows a normal behavior below $\rho \sim 1.05 \mathrm{~nm}^{-3}$ but rapidly decreases above this density, suggesting that the fluid phase becomes unstable. The virial pressures obtained along the step-wise decreasing density starting with fcc solid at $\rho$ $=1.30 \mathrm{~nm}^{-3}$ are also shown in Fig. 1 and both results are in good agreement with each other above $\rho=1.10 \mathrm{~nm}^{-3}$. The MSD becomes larger with decreasing density and tends to diverge in the region where the virial pressure shows a rapid decrease with density. The computed pressure is not constant between the freezing and melting points (the solid circles in Fig. 1) expected in the infinite (bulk) system and actually obtained by a full free-energy analysis. ${ }^{10}$ Such a behavior of the pressure is also found in other cases as exemplified for the liquid-vapor transition of the Lennard-Jones system ${ }^{1}$ and can be interpreted as the finite-size effect inevitable in any computer simulation for the phase transition. At $2100 \mathrm{~K}$ the transition from a fluidlike state to a fcc solid is actually observed at $\sim 1.05 \mathrm{~nm}^{-3}$ and the coexistence hardly occurs as 

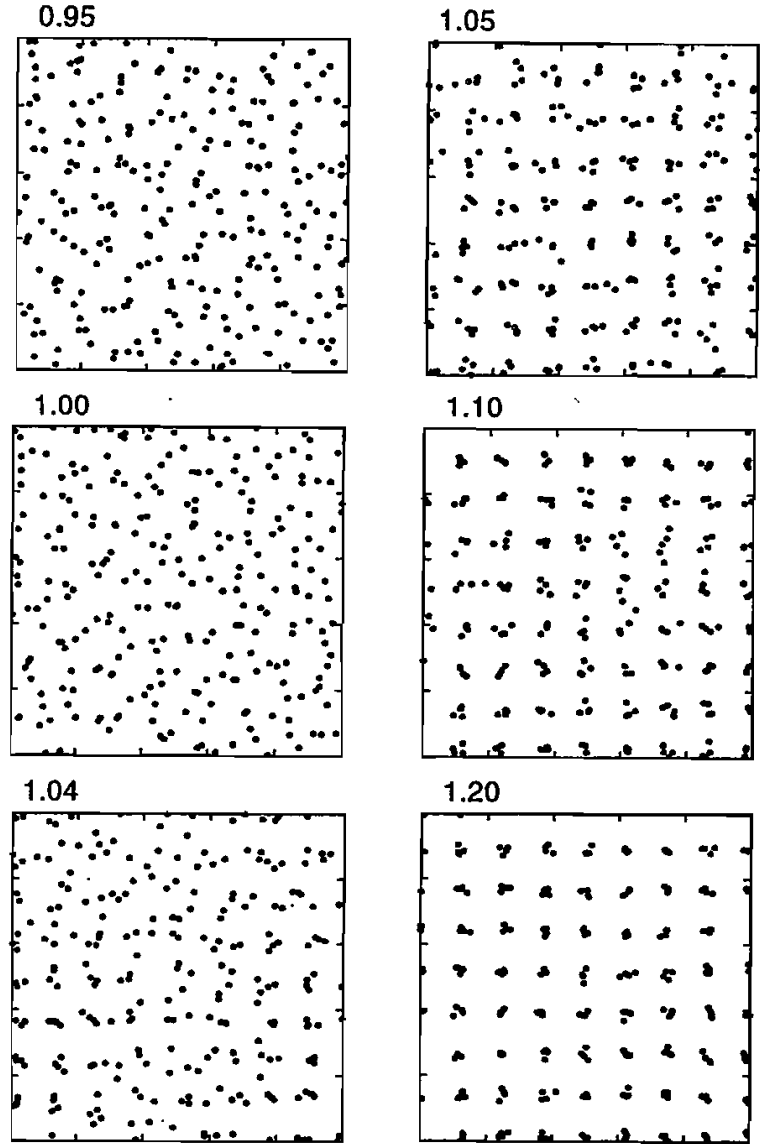

1.20

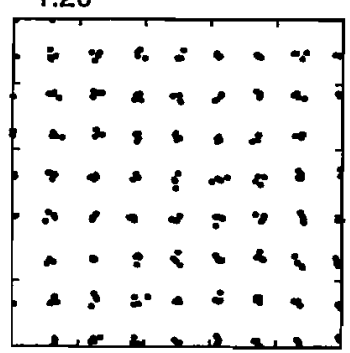

FIG. 2. Projection on the $x y$ plane of the positions (centers) of $\mathrm{C}_{60}$ molecules in the final configuration of $1 \times 10^{4} \mathrm{MCS}$ at each density at $2100 \mathrm{~K}$ : The parameter characterizing each graph is the number density $\rho$ in units of $\mathbf{n m}^{-3}$.

illustrated in Figs. 1 and 2. We note that the fluid and solid involved in this transition are something like a super-cooled fluid and super-heated solid, respectively, both being metastable in the bulk system. If we locate the "freezing point" by the above observation as Cheng et al. ${ }^{7}$ and Abramo and Coppolino ${ }^{17}$ have actually done, it falls at much higher density $\left(\sim 1.05 \mathrm{~nm}^{-3}\right)$ than the bulk value (the solid circle) determined by a full free-energy analysis. ${ }^{10}$

The present MC simulation results for the virial pressure at several temperatures and corresponding configurational energy $u_{\mathrm{ex}}$ are shown in Figs. 3 and 4, respectively. We find in Figs. 1 and 3 that the scatter of pressure is quite large, especially in the region where the system becomes unstable, and is sensitive to the way in which simulation is performed. The scatter of the computed pressure is partly due to the statistical uncertainty in simulations, which is much more serious for $\mathrm{C}_{60}$ because of its highly repulsive intermolecular potential than for ordinary systems such as the LennardJones one. ${ }^{10}$ On the other hand, the MC results for $u_{\text {ex }}$ show relatively small fluctuation, which was in fact the primary reason why we took the energy route in calculating the free energy of the fluid $\mathrm{C}_{60}$ in the previous $\mathrm{MC}$ simulation study of the phase diagram. ${ }^{10}$ We note that the computed $u_{\mathrm{ex}}$ is quite linear in $\rho$ at low and high densities, respectively, and its slope changes in the region where the pressure shows a

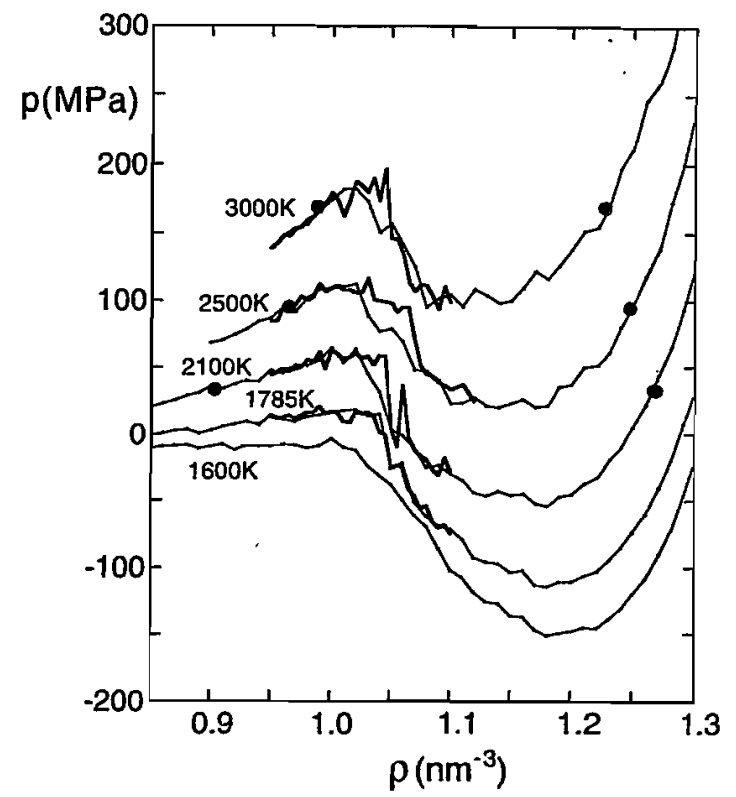

FIG. 3. MC simulations results for the virial pressure isotherms (lines): The thin line for $2100 \mathrm{~K}$ is the same as that in Fig. 1 and those for other temperatures were obtained in a similar way; the thick lines show the results obtained along the step-wise increasing densities with a smaller increment of $\Delta \rho=0.005 \mathrm{~nm}^{-3}$. The solid circles show the solid-fluid phase boundaries obtained by a full free-energy analysis (Ref. 10).

rapid decrease with density and the "freezing" begins to occur (Fig. 2). Such a feature of $u_{\text {ex }}$ has also been observed by Abramo and Coppolino in their MD simulations. ${ }^{17}$ These observations allow us to systematically determine the "freezing line" by the intersection of the straight lines interpolating the computed $u_{\mathrm{ex}}$ at low and high densities. The "freezing line" obtained in this way is shown in Fig. 5 (dashed line) and found to be in good accordance with the

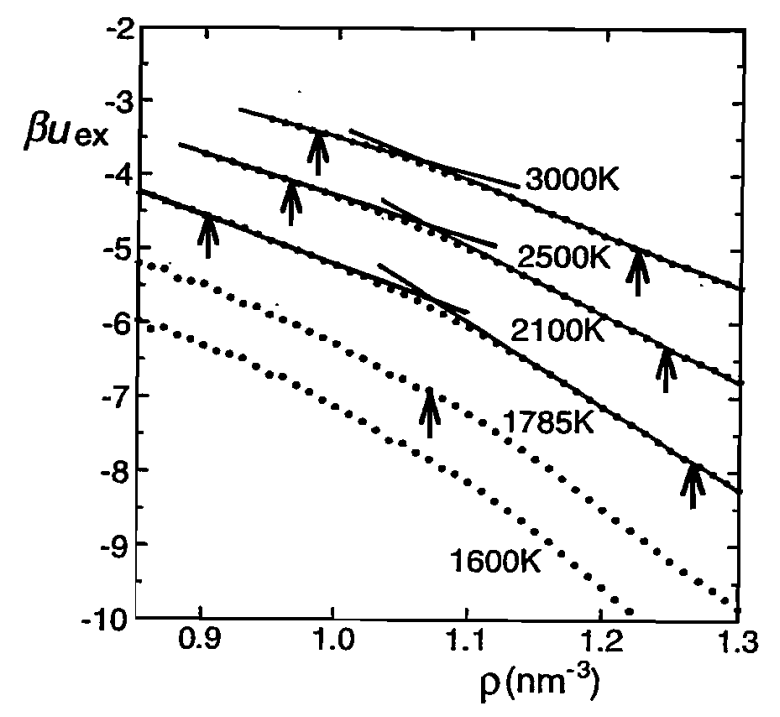

FIG. 4. MC simulation results for the configurational energy (dots): The straight lines are the linear interpolations of the results at low and high densities, respectively. The vertical arrows show the freezing point (left) and melting point (right) at each temperature (corresponding to the solid circles in Fig. 3) obtained by a full free-energy analysis (Ref. 10); the vertical arrow for $1785 \mathrm{~K}$ is the position at which the solidlike structure appeared in the MD simulation (Ref. 17). 


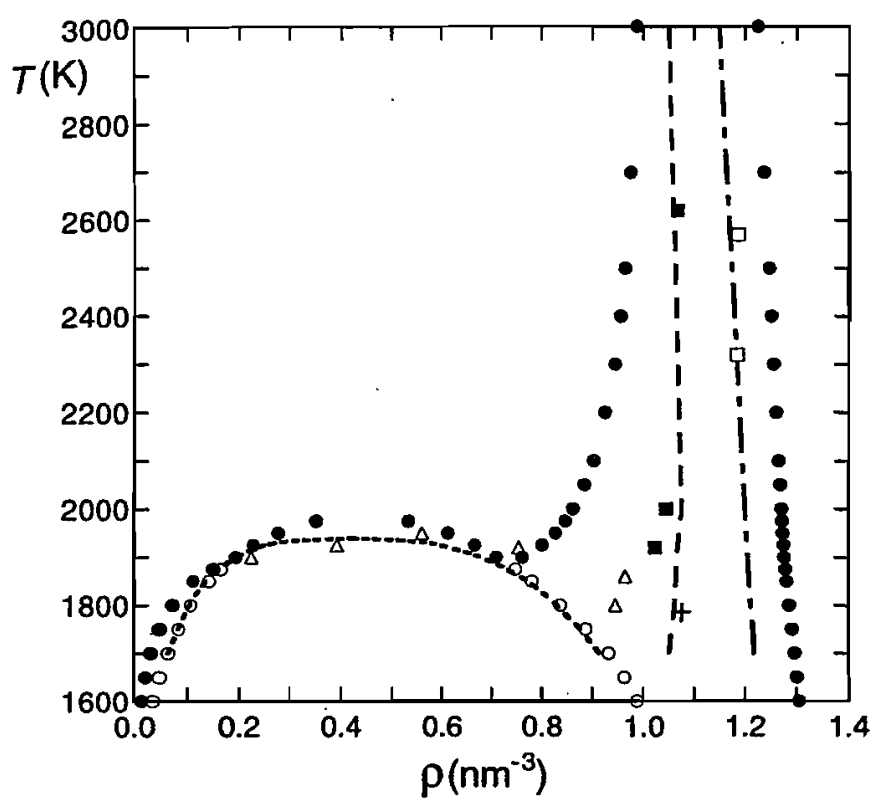

FIG. 5. High-temperature phase diagram of a model $\mathrm{C}_{60}$ : The solid and open circles represent the stable and metastable phase boundaries, respectively, obtained by a full free-energy analysis (Ref. 10); the dotted curve is the smooth interpolation of the GEMC results for the liquid-vapor binodal points (Ref. 8). The triangles, solid squares, and open squares show the liquid-vapor binodal points, freezing points, and melting points, respectively, observed in the MD simulations of Ref. 7 . The cross represents the freezing point observed in the MD simulations of Ref. 17. The dashed and chain curves represent the "freezing line" and "melting line," respectively, obtained in the present MC simulations (see the text).

corresponding MD results of Cheng $e t a l^{7}$ and Abramo and Coppolino. ${ }^{17}$ All these results are certainly on the highdensity side of the freezing line of the bulk system determined by a full free-energy analysis. ${ }^{10}$

As we have discussed in the above the solid-fluid coexistence is hardly observed in our MC simulations and the transition occurs in a very narrow range of density at a given temperature. In fact, if we assign the "melting point" to the state point at which the MSD begins to show a divergence, its density is given by $\sim 1.07 \mathrm{~nm}^{-3}$ at $2100 \mathrm{~K}$ (see Fig. 1), which is slightly higher than the "freezing density," $\rho$ $\sim 1.05 \mathrm{~nm}^{-3}$, determined by the visual method. We also tried another assignment in which the "melting point" is taken to be the state point at which the solid phase begins to show an absolute instability. At $2100 \mathrm{~K}$ the pressure shows a normal behavior above $\sim 1.19 \mathrm{~nm}^{-3}$ but below this density the system becomes unstable, i.e., the compressibility becomes negative (see Fig. 1). The "melting line" determined by this criterion is shown in Fig. 5 (the chain line). The result is consistent with that of Cheng et al. ${ }^{7}$ but we cannot trace the reason for this coincidence.

Cheng et $\mathrm{l}^{7}{ }^{7}$ have also explored the liquid-vapor phase transition of $\mathrm{C}_{60}$ by carrying out constant-volume MD simulations. They have determined the liquid-vapor binodal line using the time-dependent trajectory plots and the change of configurational energy, which behaves differently as a function of temperature in the single fluid phase and in the liquid-vapor coexistence region. Their results obtained in this manner are shown in Fig. 5 (the open triangles) and found to be in reasonable agreement with the GEMC results ${ }^{8}$ and a full free-energy analysis ${ }^{10}$ at low densities. We also found that the slope of the computed $u_{\mathrm{ex}}$ as a function of density shows a slight change near the density corresponding to the liquid-vapor phase boundaries predicted by a full freeenergy analysis. We note that the change in slope in this case is much smaller than those found for the solid-fluid transition (see Fig. 4). These results suggest that the configurational energy may be used as an indicator sensitive to the phase coexistence provided that it is computed accurately and a careful analysis is made.

Cheng et al. have also employed a block density distribution technique to locate the liquid-vapor binodal line and to confirm the above results. In this method the simulation box is divided into smaller blocks and the density in each block is estimated using constant-volume simulation data. The probability distribution of the block density would have two prominent peaks corresponding to the coexistence liquid and vapor, respectively, if the system is in the coexistence region. The two peaks would eventually merge into one on increasing temperature, suggesting that the system has reached a one-phase region. The liquid-vapor binodal line actually obtained by Cheng et al. in this way is shifted to lower temperature by $100 \mathrm{~K}$ or more and located well inside the coexistence region expected for the bulk system and determined by the GEMC and a full free-energy analysis (Fig. 5)). This result of Cheng et al. can be understood as the consequence of non-negligible interfacial energy inevitable in the finite system as in the case of the solid-fluid coexistence. The visual method in computer simulations is also not useful for locating the liquid-vapor phase boundary.

In conclusion, we have explicitly shown for the specific case of $\mathrm{C}_{60}$ that the visual appearance or disappearance of solidlike structure in computer simulations does not provide a good method for determining the solid-fluid phase boundary. The "freezing line" determined by this method certainly falls in the solid-fluid coexistence region expected for the bulk system, leading to the triple point which is much lower than the true value. In fact, the $\mathrm{MD}$ simulation result of Cheng et al. ${ }^{7}$ for the solid-fluid phase boundaries of $\mathrm{C}_{60}$ should be interpreted as an artifact characteristic to a finite system, which resolves part of controversies among the previous results for the high-temperature phase behavior of $\mathrm{C}_{60}{ }^{6-10}$ Abramo and Coppolino are also wrong in the interpretation of their MD simulations for $\mathrm{C}_{60} .{ }^{17}$ The "melting line" determined by Cheng et al. also lies deep inside the solid-fluid coexistence region expected for the bulk system and is found to coincide with the state points at which the solidlike structure begins to show absolute instability.

\section{ACKNOWLEDGMENTS}

This work has been financially supported by the Grantin-Aid for Scientific Research under Grant No. 10640360. Part of this work was carried out under the Visiting Researchers Program of the Institute for Materials Research (IMR), Tohoku University. We thank the Information Processing Center of Iwate University and the supercomputing facilities of the IMR for providing us with computer time. 
'See, e.g., D. Frenkel and B. Smith, Understanding Molecular Simulations (Academic, San Diego, 1996).

${ }^{2}$ M. de Koning, A. Antnelli, and S. Yip, Phys. Rev. Lett. 83, 3973 (1999), and references therein.

${ }^{3}$ A. Z. Panagiotopoulos, Mol. Phys. 61, 813 (1987).

${ }^{4}$ W. Krätschmer, L. D. Lamb, K. Fostiropoulos, and D. R. Huffman, Nature (London) 347, 354 (1990).

${ }^{5}$ N. W. Ashcroft, Europhys. Lett. 16, 355 (1991); Nature (London) 365, 387 (1993)

${ }^{6}$ M. H. J. Hagen et al., Nature (London) 365, 425 (1993).

${ }^{7}$ A. Cheng, M. L. Klein, and C. Caccamo, Phys. Rev. Lett. 71, 1200 (1993).

${ }^{8}$ C. Caccamo, D. Costa, and A. Fucile, J. Chem. Phys. 106, 255 (1997).
${ }^{9}$ J. Q. Broughton, J. V. Lill, and J. K. Johnson, Phys. Rev. B 55, 2808 (1997).

${ }^{10}$ M. Hasegawa and K. Ohno, J. Chem. Phys. 111, 5955 (1999).

${ }^{11}$ L. Mederos and G. Navascués, Phys. Rev. B 50, 1301 (1994).

${ }^{12}$ C. Caccamo, Phys. Rev. B 51, 3387 (1995).

${ }^{13}$ M. Tau, A. Parola, D. Pini, and L. Reatto, Phys. Rev. E 52, 2644 (1995).

${ }^{14} \mathrm{M}$. Hasegawa and K. Ohno, Phys. Rev. E 54, 3928 (1996).

${ }^{15}$ M. Hasegawa and K. Ohno, J. Phys.: Condens: Matter 9, 3361 (1997).

${ }^{16}$ L. A. Girifalco, J. Phys. Chem. 96, 858 (1992).

${ }^{17}$ M. C. Abramo and G. Coppolino, Phys. Rev. B 58, 2372 (1998).

${ }^{18} \mathrm{~J}$. D. Axe, S. C. Moss, and D. A. Neumann, in Solid State Physics: Advances in Research and Application, edited by $\mathrm{H}$. Ehrenreich and $\mathrm{F}$. Spaepen (Academic, New York, 1994), Vol. 48, p. 149. 